\title{
FUNCIONAMIENTO COGNITIVO Y FÍSICO EN ADULTAS MAYORES QUE PARTICIPAN EN UN PROGRAMA DE TAEKWONDO
}

\author{
Pedro Carazo Vargas, Carlos Ballestero Umaña, y Gerardo Araya Vargas \\ Escuela de Educación Física y Deportes \\ Universidad de Costa Rica, San José, Costa Rica \\ E-mail: pcarazov@costarricense.cr
}

\begin{abstract}
Resumen
Carazo Vargas, P., Ballestero Umaña, C., y Araya Vargas, G. (2002). Funcionamiento cognitivo y físico en adultas mayores que participan en un programa de Taekwondo. Revista de Ciencias del Ejercicio y la Salud, 2(1), 1-13. Con el propósito de analizar los efectos de un programa de taekwondo en una población de adultas mayores sobre la memoria, el tiempo de reacción, la agilidad, la fuerza y la flexibilidad se conformaron dos grupos experimentales (principiantes y avanzados), los cuales recibieron un programa de taekwondo durante diez semanas, con una frecuencia semanal de dos sesiones de 75 minutos cada una. A la vez se estableció un tercer grupo que no realizaba ningún tipo de ejercicio físico, el cual cumplió la función de control. Los tres grupos fueron medidos en las variables estudiadas al iniciar y al finalizar el tratamiento. Los resultados mostraron que los individuos pertenecientes al grupo de los avanzados poseían un nivel inicial significativamente mejor que los demás grupos en cada una de las variables, tanto físicas como cognitivas. Se dieron mejoras en las distintas variables en los dos grupos que recibieron el tratamiento (taekwondo), de una manera marcada en los sujetos principiantes, mientras que los sujetos que no realizaron actividad física (grupo control) no presentaron cambios significativos. Además se presentaron correlaciones significativas entre los porcentajes de cambio de la agilidad y la memoria y entre agilidad y la fuerza de las participantes del grupo de las principiantes, lo cual apoya la teoría de la presencia de cambios en el funcionamiento cognitivo relacionados a los cambios en el acondicionamiento físico. La práctica del taekwondo generó importantes incrementos en el desempeño físico y cognitivo con lo cual la capacidad para desarrollar las diferentes actividades de la vida diaria de las adultas mayores participantes podría verse aumentada significativamente, por lo que se considera a esta disciplina deportiva como una provechosa alternativa para que el adulto mayor obtenga importantes beneficios en su calidad de vida. PALABRAS CLAVES: Adulto mayor, taekwondo, capacidad funcional, fuerza, flexibilidad, agilidad, memoria visual, tiempo de reacción.
\end{abstract}

\section{INTRODUCCIÓN}

La limitación funcional para ejecutar las actividades del diario vivir representa un serio problema para el adulto mayor. Estas limitantes son un indicador de riesgo para la salud del individuo y afectaran directamente su calidad de vida. Morey, Pieper y CoroniHuntley (1998), describen que las limitaciones funcionales son un intermediario entre las patologías, los factores de riesgo y el deterioro, con el comienzo de la inhabilidad para valerse por sí mismo.
Estas limitaciones disminuyen, por tanto, la capacidad funcional, que se define como "la aptitud para ejecutar eficientemente las actividades básicas e instrumentales de la vida diaria” (Saliba, Orlando, Wenger, Hays y Rubenstein, 2000, p. 750).

Dentro de este contexto, se puede entender a las actividades básicas como a los comportamientos que las personas deben realizar para cuidar de sí mismas y vivir de forma independiente y autónoma. Incluyen actividades tales como bañarse, vestirse, alimentarse, movilizarse, controlar los esfínteres y el arreglo personal entre otras. 
Por su parte las actividades instrumentales de la vida diaria se refieren a acciones complejas asociadas con la vida independiente, tales como cocinar, limpiar, tomar medicamentos, usar transporte público, utilizar escaleras y realizar trabajos exteriores como jardinería y cuido de animales entre otras labores. Según Méndez (1998), la disminución de la capacidad para efectuar las actividades básicas e instrumentales de la vida diaria constituyen una de las áreas deficitarias en la edad avanzada, que no solo tiene como consecuencia la pérdida de autoestima, sino que conducen a la institucionalización de la persona mayor cuando carece de apoyos suficientes en el seno de su hogar o comunidad.

Una serie de investigaciones se han desarrollado con el objetivo de maximizar la aptitud del adulto mayor para desempeñarse satisfactoriamente en el medio y mejorar su calidad de vida y en dichos estudios se ha demostrado la capacidad de los componentes de la aptitud física de ser modificados mediante diferentes actividades corporales, con lo cual se logra un significativo aporte en el mejoramiento de la capacidad para realizar las actividades cotidianas. (Ballestero, 1999; Beissner, Collins, y Holmes, 2000; Hirvensalo, Rantanen, y Heikkinen, 2000; Morey et al., 1998; Nichols, Hitzelberger, Sherman y Patterson, 1995)

La fuerza, según Beissner y col. (2000), es uno de los componentes de la aptitud física que comprometen la habilidad funcional del individuo, pues el empeoramiento músculo esquelético produce limitaciones funcionales que contribuyen a la incapacidad, ya que este es elemento primordial para realizar muchas de las actividades de la vida diaria.

"En el adulto mayor el empeoramiento de la movilidad y la inactividad física, son factores de riesgo para una futura incapacidad $y$ muerte". (Hirvensalo y col 2000, p.493). Nichols y cols. (1995), demostraron cómo mediante un entrenamiento de fuerza, los adultos mayores residentes en un hogar para ancianos pueden incrementar su habilidad para desplazarse, subir gradas y mantener el balance, mejorando su habilidad para desarrollar las actividades de la vida diaria.

No menos importante es el deterioro que se da en la flexibilidad pues "aparte de los problemas de dolores de espalda, la flexibilidad puede limitar considerablemente la amplitud de los movimientos corporales y muchos de estos movimientos desempeñan un importante papel al realizar las tareas de la vida diaria, por lo que la pérdida de flexibilidad en edades avanzadas puede reducir considerablemente la independencia” (Meléndez, 2000, p. 33).

"En cuanto a los aspectos psicológicos del envejecimiento, se resaltan los cambios cognitivos y trastornos afectivos propios de la vejez” (Rojas, 1997, p. 34).

Becerro, Frontera y Santoja (1999), exponen cómo preferentemente a edades avanzadas se presentan una serie de alteraciones a nivel del sistema nervioso central que se colocan bajo el término global de deterioro cognitivo, lo cual tiene un importante impacto en la capacidad funcional del adulto mayor, pues disminuyen de gran manera su sentido de bienestar.

Con el envejecimiento, según Timiras (1997), se producirán modificaciones en la plasticidad neural, que alterarán la capacidad para recordar y con ello variarán las funciones cognitivas normales y la independencia de los individuos.

$\mathrm{Si}$ bien es cierto que con la edad se presentan una serie de cambios de orden cognitivo, existe amplia evidencia del provecho que significa la actividad física para el adulto mayor. El ejercicio, según Shephard (1995), representa beneficios importantes en la calidad de vida de los adultos mayores, pues tiene una particular importancia en el mantenimiento de la capacidad para ejecutar las actividades de la vida diaria y conservar la independencia, pues puede proveer positivos cambios en el funcionamiento mental y físico. Esta posición es ratificada por Etnier, Salazar, 
Landers, Petruzzello, Han, y Nowell (1997), que mediante el meta-análisis Influencia del estado físico y el ejercicio en el funcionamiento cognitivo, concluyeron que el acondicionamiento y la actividad física poseen un efecto positivo en el desempeño cognitivo, condición que se presenta especialmente cuando el ejercicio es administrado bajo un tratamiento crónico, en individuos sedentarios.

El anterior estudio coincide con lo hallado por Sanabria (1995), que efectuó un meta-análisis sobre los efectos del ejercicio en parámetros cognitivos, encontrando que existe un efecto significativo del ejercicio sobre la inteligencia, la creatividad, el tiempo de reacción y especialmente sobre la memoria. Estas mejorías se presentan tanto con ejercicio agudo como crónico, siendo más considerable el efecto alcanzado con la práctica del ejercicio crónico, destacándose la presencia de mayores tamaños de efecto cuando los individuos tienen entre 46 y 60 años.

Esta tesis refuerza lo descrito por Marqués (1993), que concluyó que tanto la capacidad intelectual, la inteligencia, como la memoria, pueden ser optimizados en el adulto mayor, pero para esto precisan de un nivel adecuado de actividad específica que estimule estas áreas directamente. Sanabria (1995) amplifica esta posición, indicando que las mejoras más representativas en la inteligencia y en la memoria se dan cuando el ejercicio es de tipo aeróbico. No obstante se debe de tomar en cuenta de que han sido pocos los estudios que han examinado las actividades no aeróbicas, un área donde según Sanabria (1995), los principales efectos se han mostrado en el tiempo de reacción, por lo cual el análisis de diferentes modalidades de ejercicio cobra gran relevancia en vías de evaluar esta tesis.

En la actualidad el taekwondo es una actividad deportiva que ha adquirido un gran desarrollo, esta modalidad utiliza un metabolismo energético mixto y por la actividad que conlleva representa una alternativa para la mejora de la capacidad cognitiva y física, ya que "el taekwondo es ante todo una disciplina mental, corporal y espiritual. Las manifestaciones físicas en formas de ataques no son más que la parte visible” (Yadory, 1997, p. 108). El taekwondo es un arte marcial coreano, es tanto una manera de golpear y patear como una forma de vida. Ballestero (1999), define esta forma de vida (conocida como "Do" entre los practicantes de artes marciales), como un camino de transformaciones interiores que pretenden la realización y perfeccionamiento personal, donde el individuo busca auto disciplinarse en lo físico, moral, espiritual y emocional.

En Costa Rica los estudios en el área de las artes marciales con una población de adultos mayores han sido escasos. Ballestero (1999) analizó la calidad de vida, la autoestima y el estado anímico y físico, de adultas mayores por medio de un programa de Karate Do. Para llevar a cabo este estudio se sometió a 21 mujeres mayores de 60 años al tratamiento, que se efectuó en dos sesiones semanales durante 10 semanas. Al finalizar el periodo de prácticas, se observó aumentos significativos en la flexibilidad, la fuerza, la autoestima y el vigor, así como descensos en la tensión, la depresión y la fatiga. Además, las participantes manifestaron haber optimado su autonomía e independencia, lo que les permitió disfrutar de una vida más placentera consigo mismo y con las personas que las rodean, alcanzando de este modo un ambiente propicio en su vida personal, familiar y comunal.

Carazo, Ballestero y Salazar (2000), le dieron seguimiento a este primer estudio, encontrando que las personas participantes en el programa inicial que continuaron su entrenamiento en artes marciales presentaban una tendencia a la mejoría en la fuerza, la flexibilidad, la autoestima y el estado de ánimo, mientras que los individuos que se retiraron del programa experimentaron reducciones en las ganancias previas.

Si bien en diversos estudios se ha comprobado la importancia de la actividad física en parámetros de preponderante 
importancia para la calidad de vida de las personas mayores, al pretender evaluar la capacidad funcional como tal, su determinación no siempre se ha establecido de la manera idónea. Beissner y cols. (2000), exponen que muchas de las investigaciones son enfocadas en la decadencia funcional y la incapacidad para realizar diferentes actividades lo cual brinda resultados inexactos para una gran cantidad de adultos mayores.

Los indicadores de salud deben expresarse no solo en términos de enfermedad o patología sino en términos de actividad funcional o vitalidad.

Tomando en cuenta que Morel (1996), estableció la existencia de asociación entre la condición física y la capacidad funcional de los adultos mayores, se estima conveniente el determinar los componentes de la aptitud física que inciden directamente sobre la capacidad funcional, con el fin de lograr una valoración más efectiva del adulto mayor independiente funcionalmente.

Biddle (1995), sostiene que aunque las revisiones narrativas del efecto del ejercicio en la cognición, proveen un respaldo para los beneficios de la actividad física, esta área aún no está totalmente clara, pues los mecanismos de medición de dichos parámetros no siempre han sido los óptimos.

Aunado a este elemento surgen una serie de factores que fundamentan la importancia de la presente investigación, tales como el estudio de diferentes modalidades de actividad física, ya que representan un medio imprescindible para llevar a cabo una eficiente evaluación de los resultados, en este aspecto el taekwondo por sus características energéticas y por las exigencias tanto en el ámbito físico como cognitivo que requiere gran parte de sus destrezas, constituye una alternativa importante de estudiar en procura de fortalecer la capacidad funcional del adulto mayor.

Aunque Sanabria (1995), analizó la duración óptima de los programas de actividad física, estableciendo que para obtener ganancias a nivel cognitivo dicha duración está entre los 90 y 120 días, es importante examinar los efectos del ejercicio en parámetros cognitivos, en individuos que han permanecido realizando una actividad durante un tiempo más prolongado.

Los individuos entrenados poseen características especiales que los diferencian de los que no realizan actividad física. Weicker (1996), afirma que el acondicionamiento físico prolongado permitirá modificaciones importantes en el organismo de los individuos, destacándose los cambios en la composición corporal, en el músculo esquelético y en el potencial metabólico, aspectos que mediante el entrenamiento irán diferenciando a los individuos sedentarios de los físicamente activos.

Los individuos que han recibido un entrenamiento previo han atravesado un proceso que les ha permitido obtener diferentes ganancias y mejorar su rendimiento físico, en este aspecto es importante aclarar si las personas mayores que ya están acondicionadas físicamente pueden seguir mejorando sus capacidades físicas y cognitivas.

Aunque existe una tendencia a que la mejora en la condición física repercute positivamente en el desempeño de las actividades cognitivas, "muchos estudios no reportan los cambios físicos logrados, lo que ha dificultado concluir a favor de esta teoría” (Sanabria, 1995, p. 23). Así mismo hay controversia sobre si es el acondicionamiento físico, el responsable de las mejoras cognitivas debido al ejercicio (Etnier y cols., 1997) y si es así, qué componente físico es el más importante mejorar, para lograr beneficios cognitivos, o si bien es más importante acondicionar integralmente a los sujetos.

De acuerdo a Hess y Blanchard (1999), los individuos mayores enfrentan una serie de problemas debido al descenso de sus capacidades, no obstante, los mecanismos utilizados para enfrentar esta situación son escasos. Por lo cual, la aclaración de 
diversas circunstancias que interfieren en la mejoría cognitiva y la determinación de nuevas alternativas para optimizar la capacidad funcional son elementos de suma importancia en procura de contribuir a mejorar la calidad de vida del adulto mayor. Buscando este fin la presente investigación se plantearon dos objetivos. Primero, analizar los efectos de un programa de taekwondo en el área cognitiva (memoria visual, tiempo de reacción) y el área física (fuerza, flexibilidad, agilidad) tanto en adultas mayores sedentarias como en previamente entrenadas. Segundo, correlacionar las modificaciones en el área física y cognitiva, producto de la práctica del taekwondo en adultas mayores.

\section{METODOLOGÍA}

\section{Sujetos}

La muestra de esta investigación se conformó de mujeres con edades comprendidas entre los 58 y 77 años, capaces de movilizarse por sí mismas, con aprobación médica mediante un dictamen, que no posean ninguna contraindicación para realizar actividad física. Se contó con un total de 37 individuos divididos por conveniencia en tres grupos. El grupo experimental A estuvo compuesto por 15 mujeres, que no participaban desde un mínimo de un año de ningún programa de actividad física. El grupo experimental B lo conformaron 10 mujeres que tenían un promedio de 2 años de practicar taekwondo bajo características similares a las que se emplearon en los procedimientos de la presente investigación. El grupo control estuvo compuesto por 12 mujeres que no realizaban ningún tipo de actividad física, las cuales eran miembros de un club de coro de adultos mayores que se reúnen una vez a la semana para ensayar.

\section{Instrumentos de medición}

La flexibilidad y la agilidad, se estimaron mediante las pruebas para adultos mayores, diseñadas y validadas por la American Alliance for Health, Physical Education, Recreation and Dance (AAHPERD) (Clark, 1989).

En la prueba de agilidad se toma el tiempo que tarda el sujeto en efectuar un recorrido en el cual se debe realizar cambios de dirección y sentarse periódicamente.

En la prueba de flexibilidad se determina la movilidad a nivel coxofemoral, donde el sujeto debe de sentarse en el suelo con las piernas separadas y efectuar el mayor desplazamiento posible hacia delante manteniendo ambas manos siempre rozando el suelo.

La fuerza se determinó mediante la prueba 30" Chair Stand (Jones, Rikli y Beam, 1999), que posee una confiabilidad de 0.92 y una validez de 0.71 . Durante la prueba la persona debe de comenzar sentada y ponerse de pie la mayor cantidad de veces posible durante 30 segundos.

La memoria se analizó mediante el Test Verbal Script Digit Span (Solera 2000), el cual fue modificado bajo el criterio de expertos para realizarlo de manera visual. En la prueba el evaluador le enseñó al ejecutante una tarjeta con una secuencia de números, que permaneció visible tantos segundos como dígitos tenía la tarjeta. Al finalizar el lapso de tiempo la tarjeta se ocultaba y el individuo debía repetir la secuencia de números en el mismo orden. En cada nivel el individuo contaba con dos intentos distintos, si el sujeto no se equivocaba pasaba al siguiente nivel. El primer nivel iniciaba con tres números $\mathrm{y}$ luego se aumentaba un número en cada nivel. El tiempo de reacción fue obtenido mediante el Test de la Mano de Nelson, el cual posee un coeficiente de confiabilidad de 0.79 (Solera, 2000). El sujeto apoyaba su antebrazo en una mesa y colocaba sus dedos pulgar e índice formando una "pinza" a nivel del cero de una regla que era sostenida por el 
evaluador. La regla era soltada y el individuo debía tomarla lo más pronto posible. Se contó con cinco intentos de práctica y veinte que fueron registrados, de los cuales se eliminaron los cinco resultados más bajos y los cinco más altos para tomar el promedio con los resultados restantes.

\section{Procedimiento}

Ambos grupos recibieron un programa durante 10 semanas con 2 sesiones por semana, de una duración de 75 minutos cada una.

El programa consistió en el análisis de los fundamentos filosóficos $y$ en el aprendizaje de destrezas de bloqueos, patadas y golpes del taekwondo, por parte de los participantes, de acuerdo a su nivel (principiantes o avanzados. Cada una de las sesiones se compuso de tres elementos básicos, la lectura y comentario de una reflexión durante 5 minutos aproximadamente, la realización de ejercicios de calentamiento durante 10 minutos donde se realizaban ejercicios aeróbicos y de flexibilidad, también se desarrollaron actividades acondicionamiento físico durante aproximadamente 15 minutos, además del aprendizaje y repaso de alguna destreza. Durante las primeras 5 semanas del programa, el trabajo se enfocó a la enseñanza de destrezas y durante las últimas 5 semanas al aprendizaje de una forma (secuencia de movimientos que simulan un combate imaginario). Ambos grupos experimentales realizaron el mismo trabajo a nivel físico, cada grupo aprendió las destrezas diferentes de acuerdo a su nivel, pero siempre se busco asemejar el volumen, intensidad y tipo de trabajo. El grupo control permaneció sin recibir ningún tipo de programa físico, efectuando solamente las actividades de su rutina diaria y las reuniones habituales de su grupo.

A los tres grupos se les efectuaron mediciones en las variables de estudio antes de iniciar el programa $\mathrm{y}$ al finalizar el tratamiento, en las sesiones de entrenamiento previas y posteriores a las semanas 1 y 10 . Además se realizaron mediciones de la longitud de brazos y piernas con la finalidad de descartar alguna diferencia entre los grupos que pudiera beneficiarlos durante las mediciones de flexibilidad, fuerza y agilidad.

\section{Análisis estadístico}

Los datos se analizaron mediante estadística descriptiva e inferencial. Se aplicaron análisis de varianza de dos vías (3 x 2) para grupos independientes con medidas repetidas en un factor (nivel $x$ mediciones). Se calcularon análisis de efectos simples y pruebas post hoc de Tukey cuando fue necesario. Además se obtuvieron los porcentajes de cambio en las diferentes variables dependientes y se les aplicó pruebas de correlación de Spearman para establecer las relaciones entre los mismos.

\section{RESULTADOS}

La tabla $\mathrm{N}^{\circ} 1$ describe las características corporales de los individuos participantes. Se muestran la edad y longitud de manos y piernas, como elementos capaces de influir en los resultados al comparar los grupos.

El respectivo análisis estadístico mostró que no existe diferencia significativa en ninguno de los tres factores, (edad: $F_{(2,34)}$ $=0.57 p>0.05$; longitud de brazo: $F_{(2,34)}=$ $0.70, p>0.05$; $y$ longitud de pierna: $F_{(2,34)}=$ 1.95, $p>0.05$ ) entre los grupos (experimentales y control), por lo cual se puede asegurar que esas características no fueron un factor influyente en los resultados de los análisis estadísticos posteriores.

Las tablas $\mathrm{N}^{\circ} 2$ y $\mathrm{N}^{\circ} 3$, muestran los promedios y desviaciones estándar obtenidos por los integrantes de los grupos de principiantes, avanzados y control en cada una de las variables de estudio. 
Tabla $N^{\circ} 1$. Promedios y desviaciones estándar de las características generales de los sujetos

\begin{tabular}{|c|c|c|c|}
\hline & Edad (años) & Longitud de Brazo (cm) & Longitud de Pierna (cm) \\
\hline Principiantes $(n=15)$ & $65.13 \pm 5.63$ & $72.31 \pm 2.07$ & $99.20 \pm 3.61$ \\
\hline Avanzados $(\mathrm{n}=10)$ & $64.00 \pm 5.91$ & $70.65 \pm 5.13$ & $96.30 \pm 4.76$ \\
\hline Control $(n=12)$ & $66.67 \pm 6.30$ & $71.17 \pm 2.21$ & $98.06 \pm 2.19$ \\
\hline
\end{tabular}

Según el análisis estadístico efectuado mediante ANOVA mixto A x B (3 $\mathrm{x} 2$, grupos por mediciones) se encontró una interacción significativa para la variable agilidad $\left(F_{(2,34)}=7.20 ; p<0.05\right)$. El análisis de efectos simples estableció diferencias entre las dos mediciones de los individuos principiantes $\left(F_{(1,34)}=36.22, p<0.05\right) \mathrm{y}$ entre las dos mediciones realizadas a los sujetos avanzados $\left(F_{(1,34)}=11.40, p<0.05\right)$, además también se presentaron diferencias significativas tanto entre las mediciones hechas a los grupos en el pre test $\left(F_{(1,34)}=\right.$ $225.41, p<0,05)$ como en el post test $\left(F_{(1,34)}\right.$ $=177.87, p<0.05)$. La prueba post hoc de Tukey mostró diferencias entre la primera y la segunda medición de los sujetos principiantes; entre las dos mediciones de los sujetos avanzados y las dos mediciones de los individuos del grupo control y la primera de los principiantes; además existieron diferencias entre el nivel alcanzado por los principiantes en la segunda medición y las dos mediciones del grupo control.

En el gráfico $\mathrm{N}^{\circ} 1$ se ilustran los resultados anteriores donde se observa cómo antes de iniciarse el programa, el grupo de los avanzados tenía una mejor agilidad que los sujetos de los otros grupos pues su velocidad de desplazamiento es significativamente menor. Al finalizar el programa de taekwondo, los sujetos de ambos grupos que participaron en él, lograron aumentar su agilidad disminuyendo su tiempo de desplazamiento, dándose este efecto de una manera más marcada en los individuos principiantes, mientras que en los integrantes del grupo control no presentaron cambios significativos entre mediciones.

En el gráfico $\mathrm{N}^{\circ} 2$ se presentan los resultados encontrados en la variable fuerza, donde se observa una interacción significativa $\left(F_{(2,34)}=11.42, p<0.05\right)$. El análisis de efectos simples indicó que existían diferencias en las mediciones efectuadas al grupo de los principiantes $\left(F_{(1,34)}=35.79, p<0.05\right) \mathrm{y}$ entre las dos mediciones realizadas a los sujetos avanzados $\left(F_{(1,34)}=41.76, p<0.05\right)$, mientras que en el grupo control no se presentaron diferencias significativas, adjuntamente se dieron diferencias entre las mediciones del pre test $\left(F_{(1,34)}=25.76, p<\right.$ $0.05)$ y entre las mediciones del post test $\left(F_{(1,34)}=56.85, p<0.05\right)$.

El análisis post hoc mostró que las diferencias se encontraban entre la primera y la segunda medición de los principiantes y entre la primera de los principiantes respecto a las dos mediciones de los avanzados. Además existió diferencia entre todas las mediciones de los demás grupos y la efectuada la segunda ocasión por los avanzados.

Tabla $\mathbf{N}^{\circ}$ 2. Promedios y desviaciones estándar de la agilidad, la fuerza y la flexibilidad.

\begin{tabular}{lcccccc}
\hline \multirow{2}{*}{ Grupo } & \multicolumn{2}{c}{ Agilidad (s) } & \multicolumn{2}{c}{ Fuerza (reps / 30 s) } & \multicolumn{2}{c}{ Flexibilidad (cm) } \\
\cline { 2 - 7 } \multicolumn{1}{c}{ Pre } & Post & Pre & Post & Pre & Post \\
\hline Principiantes & $28.32 \pm 4.24$ & $25.40 \pm 3.38$ & $12.60 \pm 1.99$ & $14.80 \pm 1.82$ & $67.73 \pm 7.81$ & $69.57 \pm 8.65$ \\
Avanzados & $25.35 \pm 2.40$ & $23.35 \pm 2.85$ & $14.70 \pm 2.00$ & $17.60 \pm 2.07$ & $73.90 \pm 16.62$ & $75.45 \pm 15.63$ \\
Control & $28.46 \pm 3.86$ & $28.29 \pm 3.27$ & $13.08 \pm 2.11$ & $13.25 \pm 1.76$ & $65.17 \pm 12.74$ & $65.33 \pm 12.8$ \\
\hline
\end{tabular}


Tabla $\mathbf{N}^{\circ}$ 3. Promedios y desviaciones estándar de la memoria auditiva y el tiempo de reacción.

\begin{tabular}{lcccc}
\hline \multirow{2}{*}{\multicolumn{1}{c}{ Grupo }} & \multicolumn{2}{c}{ Memoria (puntos) } & \multicolumn{2}{c}{ Tiempo de reacción (s) } \\
\cline { 2 - 5 } & Pre & Post & Pre & Post \\
\hline Principiantes (n= 15) & $3.47 \pm 1.30$ & $4.47 \pm 1.41$ & $0.30 \pm 0.21$ & $0.18 \pm 0.02$ \\
Avanzados (n= 10) & $3.90 \pm 1.29$ & $4.30 \pm 1.42$ & $0.17 \pm 0.01$ & $0.17 \pm 0.01$ \\
Control (n= 12) & $4.67 \pm 1.30$ & $4.75 \pm 0.87$ & $0.19 \pm 0.02$ & $0.20 \pm 0.02$ \\
\hline
\end{tabular}

Se observa una mejora en los niveles de memoria pues se presentó un aumento significativo en el promedio obtenido en el post test respecto al pre test, donde se puede apreciar que el aumento en el promedio de la memoria se dio debido a los mayores incrementos que desarrollaron los sujetos que practicaban taekwondo (Gráfico $\mathrm{N}^{\circ} 3$ ).

El comportamiento de los grupos permite apreciar que aunque el grupo con mayor tiempo de práctica (avanzados) poseía un nivel superior de fuerza antes de iniciar el programa, independientemente del nivel que se tenga ya sea principiante o avanzado, la participación en el programa de taekwondo produjo ganancias significativas en los niveles de fuerza de ambos grupos experimentales

En la variable memoria existió diferencia significativa entre las mediciones efectuadas a los grupos $\left(F_{(1,34)}=11.03, p<\right.$ 0.05).

En la variable tiempo de reacción existió diferencia significativa entre los grupos $\left(F_{(2,34)}=3.70, p<0.05\right)$.

En el gráfico $\mathrm{N}^{\circ} 4$ se aprecia que al inicio los sujetos principiantes tenía un tiempo de reacción mucho más lento que los otros dos grupos. No obstante mientras que los sujetos del grupo control mostraron una tendencia a aumentar su tiempo de reacción, mediante el entrenamiento recibido, los individuos principiantes mostraron una tendencia a igualar el nivel inicial de tiempo de reacción de los sujetos avanzados, pero este cambio no fue significativo.

En el gráfico $\mathrm{N}^{\circ} 5$ se muestra el comportamiento observado en la flexibilidad. Aunque no se dieron diferencias significativas sobre esta variable se destaca la tendencia de los sujetos avanzados a presentar mejores rangos de flexibilidad, así como la tendencia a evidenciar mejorarías por parte de los participantes del programa de taekwondo, con una mayor acentuación de los integrantes del grupo de principiantes.

En la tabla $\mathrm{N}^{\circ} 4$ se observan los porcentajes de cambio alcanzados en las cinco variables analizadas por los grupos de principiantes y avanzados y control, luego del periodo de tratamiento.

\section{Tabla $N^{\circ}$ 4. Porcentajes de Cambio}

\begin{tabular}{lccc}
\hline & Principiantes & Avanzados & Control \\
\hline Memoria & 28.82 & 10.26 & 1.71 \\
Tiempo de & -40.00 & 0.00 & 5.26 \\
Reacción & & -7.89 & -0.60 \\
Agilidad & -10.31 & 19.73 & 1.30 \\
Fuerza & 17.46 & 2.10 & 0.25 \\
Flexibilidad & 2.72 & &
\end{tabular}

Tabla $\mathrm{N}^{\circ} 5$. Correlaciones entre los porcentajes de cambio. Principiantes

\begin{tabular}{lccccc}
\hline & Mem. & T. R & Agil. & Fuer. & Flex. \\
\hline Mem & --- & 0.13 & $0.63^{*}$ & -0.21 & -0.16 \\
T. R. & --- & --- & 0.08 & -0.37 & -0.46 \\
Agil. & --- & --- & --- & $-0.52 *$ & -0.21 \\
Fuer. & --- & --- & --- & --- & -0.08 \\
Flex. & --- & --- & --- & --- & --- \\
\hline$* p<0.05$ & & & &
\end{tabular}

Tabla $\mathrm{N}^{\circ}$ 6. Correlaciones entre los porcentajes de cambio. Avanzados

\begin{tabular}{lccccc}
\hline & Mem. & T. R & Agil. & Fuer. & Flex. \\
\hline Mem & --- & $-0.64^{*}$ & 0.26 & -0.34 & -0.35 \\
T. R. & --- & --- & -0.14 & 0.07 & 0.33 \\
Agil. & --- & --- & --- & -0.18 & -0.11 \\
Fuer. & --- & --- & --- & --- & 0.24 \\
Flex. & --- & --- & --- & --- & --- \\
\hline$* \quad p<0.05$ & & & &
\end{tabular}


Las tablas $\mathrm{N}^{\circ} 5$ y $\mathrm{N}^{\circ} 6$ muestran las correspondientes correlaciones obtenidas entre los porcentajes de cambio presentados por los sujetos principiantes, avanzados y control en las variables estudiadas.

La correlación encontrada entre los porcentajes de cambio de la agilidad y la memoria de los sujetos del grupo de principiantes ( $r=-0.63, p<0.05$ ), indica que los sujetos que experimentaron una mejora en la prueba de agilidad, ya que lograron disminuir el tiempo requerido para completar la prueba, también presentaron un aumento en sus niveles de memoria visual (Gráfico $\mathrm{N}^{\circ} 6$ ).

Gráfico $\mathrm{N}^{\circ}$ 1. Cambios en la agilidad entre mediciones
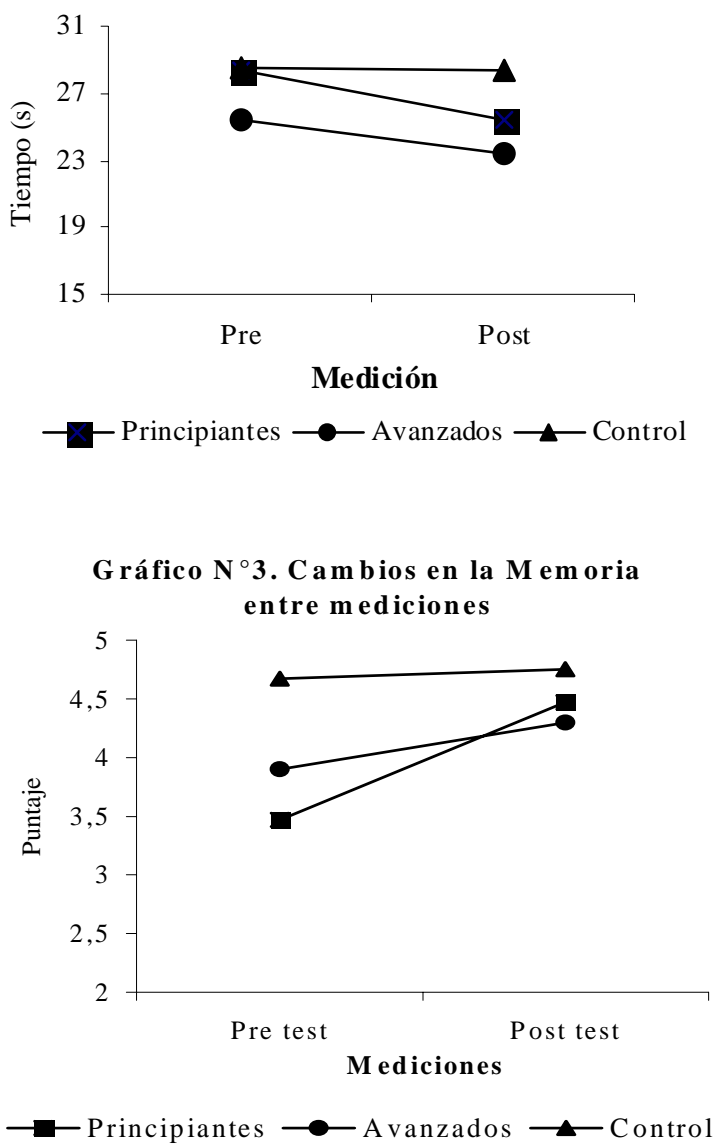

La correlación encontrada entre los porcentajes de cambio de la fuerza y la agilidad de los principiantes $(r=-0.52, p<$ $0.05)$, muestra una tendencia en la que altos porcentajes de cambio en la fuerza se asocian con cambios positivos en la agilidad pues se reducen los tiempos de desplazamiento (Gráfico $\mathrm{N}^{\circ}$ 7).

Además de estas relaciones, también se encontró una correlación entre los cambios experimentados en la memoria y el tiempo de reacción en los individuos del grupo de avanzados ( $r=-0.64, p<0.05$ ), la cual indica que a mayores niveles en la memoria, el tiempo de reacción tiende a mejorar.

Gráfico $N^{\circ}$ 2. Cambios en la fuerza entre mediciones

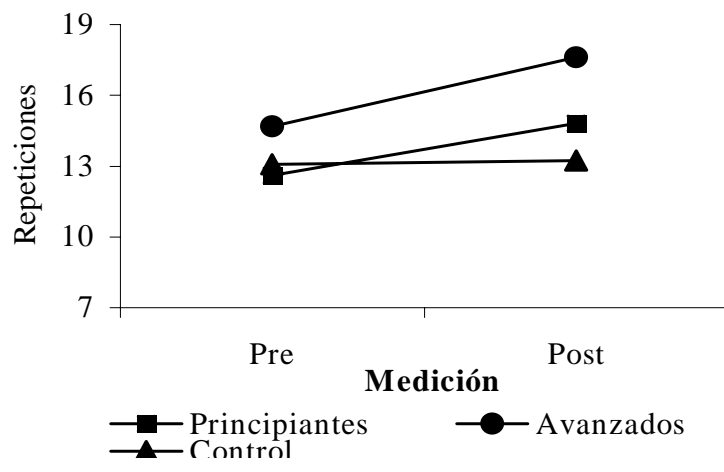

Gráfico $\mathrm{N}^{\circ}$ 4. Cambios en el Tiempo de reacción entremediciones

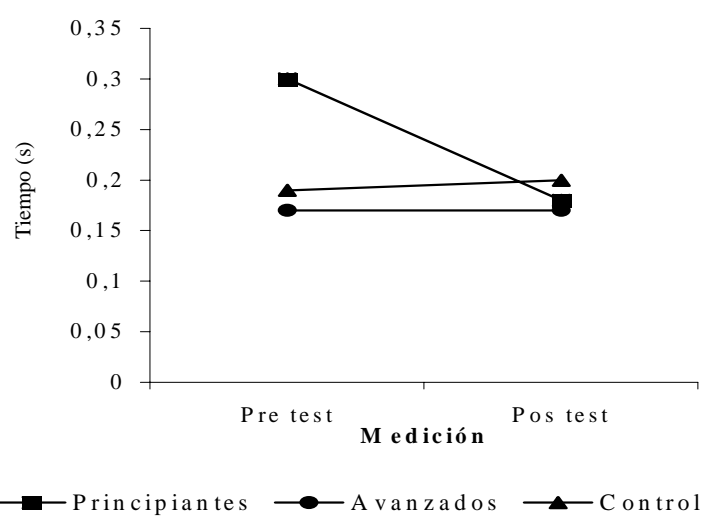


Gráfico $\mathrm{N}^{\circ} 5$. Cambios en la flexibilidad entre mediciones

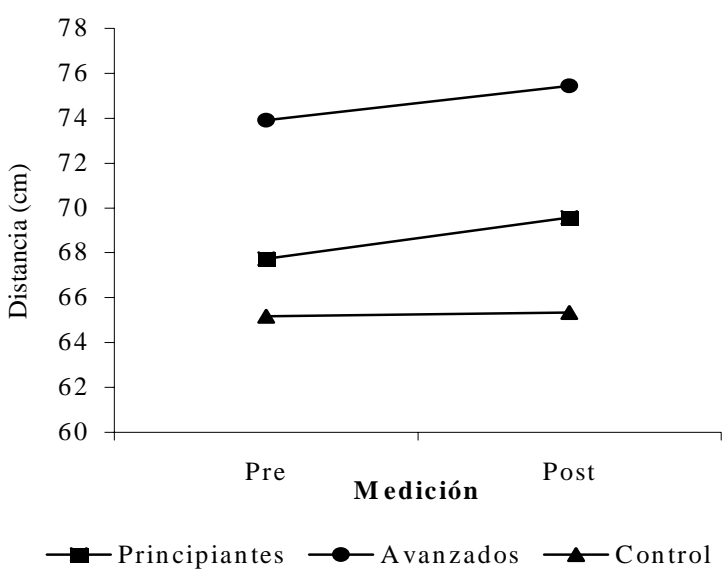

\section{DISCUSIÓN}

Tomando en cuenta que Mora (2002), describió como la actividad física contribuye al bienestar corporal y mental, mejorando la movilidad, la independencia, la estabilidad emocional y la calidad de vida en general; $\mathrm{y}$ retomando los presentes resultados; se afirma que la ejecución de actividad física es un elemento primordial para mantener una óptima capacidad funcional. En este aspecto el taekwondo surge como una provechosa alternativa para que el adulto mayor obtenga importantes beneficios de orden físico y cognitivo.

La práctica del taekwondo generó importantes mejoras en el desempeño físico, pues se apreció como los individuos avanzados presentaban mejores niveles iniciales en la fuerza, la flexibilidad y la agilidad que los sujetos principiantes, mostrando el impacto de la práctica a largo plazo del taekwondo en estos sujetos. Sin embargo, tanto los avanzados como los principiantes experimentaron mejoras, siendo estas mayores en los sujetos principiantes. Este comportamiento apoya los resultados de Morel y Salazar (1995), que al analizar la condición física de los adultos mayores, concluyeron que la práctica regular de actividad física disminuye el declive de la mayoría de los parámetros fisiológicos asociados con la salud.

La ejecución de las distintas destrezas del taekwondo requiere una gran amplitud y movilidad en las diferentes articulaciones, además en los distintos movimientos se requieren frecuentes cambios de dirección con la coordinación de diversos segmentos corporales, razones por las cuales se explican los niveles alcanzados en la flexibilidad y la agilidad. Además debido a las características de esta disciplina el empleo de las piernas es muy frecuente, por lo cual se considera a este compromiso como el responsable directo de las mejoras en la fuerza

Aunque en el análisis estadístico no se evidenciaron interacciones significativas en la flexibilidad, la memoria y el tiempo de reacción, es importante reconocer que los porcentajes de cambio de los diferentes grupos mostraron que estos tuvieron un comportamiento distinto entre sí, donde las principales mejoras se dieron en los grupos que recibieron el programa de taekwondo, presentándose una mayor magnitud en los cambios de los individuos principiantes, manifestándose de esta manera los beneficios del programa de taekwondo sobre estas variables.

Las mejoras obtenidas en el ámbito cognitivo coinciden con las halladas por Aguirre (2000), quien describió cómo los adultos mayores participantes de un entrenamiento de fuerza presentaban beneficios tanto en la memoria como en el tiempo de reacción.

Ambas mejoras deben de ser analizadas para reforzar la tesis de que "el declive cognitivo no puede ser atribuido solamente a la edad en las personas mayores saludables" (Starr, Deary, Inch, Cross y MacLennan, 1997). Sin importar la edad cronológica, el tipo de actividad que se desarrolla durante la edad avanzada es un elemento fundamental para mantener una adecuada salud mental. 


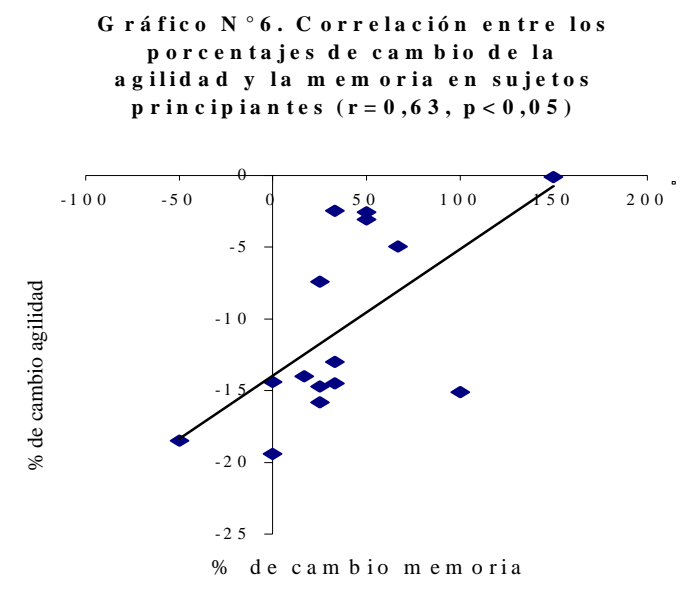

En este aspecto se mostró cómo el taekwondo representa un mecanismo apto para adquirir mejorías en la capacidad cognitiva. La práctica del taekwondo requiere de un elevado compromiso cognitivo para desarrollar muchas de las diferentes destrezas de este arte marcial, tal es el caso del aprendizaje de las "formas" que durante el programa represento un aspecto relevante para obtener las mejoras en el tiempo de reacción y la memoria.

Tomando en cuenta que Hess y Blanchard (1999), describen que a pesar de las evidencias científicas, normalmente los individuos mayores consideran el deterioro físico y cognitivo como un hecho inevitable y no toman ninguna vía para maximizar su funcionamiento.

El conocimiento desde etapas tempranas acerca de los beneficios adquiridos al mantener hábitos deportivos, representa un elemento de vital importancia para la salud de la población en general.

En nuestra sociedad comúnmente se ha considerado la práctica de artes marciales como apropiada solamente para niños y jóvenes. Por lo cual, la comprensión del aporte benéfico que el taekwondo y la actividad física poseen sobre los efectos del envejecimiento en los diferentes sistemas del organismo, permitirán que no se considere el descenso en la capacidad física para realizar

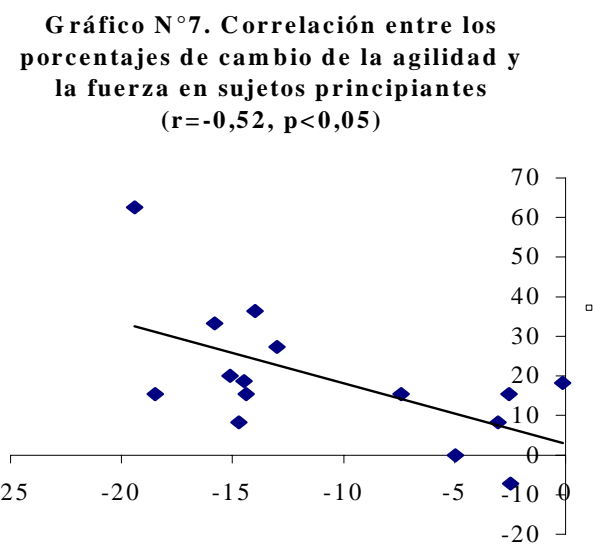

$\%$ de cambio agilidad

las actividades de la vida diaria como un hecho condicionado del envejecimiento.

Solamente la educación de los diferentes individuos integrantes de la sociedad, permitirá que cuando el sujeto llegue a una etapa de edad avanzada posea los hábitos físicos adecuados y no se presente una reducción en su calidad de vida producto del descenso de la capacidad física.

Es importante destacar cómo tanto en las variables físicas como en las de índole cognitivo, las participantes pertenecientes al grupo de avanzados poseían los niveles más altos al inicio del estudio, con lo cual se refuerza la posición que respalda los beneficios producidos mediante la práctica del taekwondo a largo plazo.

Se debe de tomar en cuenta que en la memoria el grupo control no presentó el comportamiento que de acuerdo a su función se esperaba. Estas personas pertenecían a un grupo de coro, que se reunían una vez a la semana para ensayar y conversar, aunque se estimó que la actividad efectuada por las participantes no representaba un efecto significativo sobre las distintas variables, se observó que las mismas poseían un nivel inicial de memoria superior a las participantes de los otros grupos, lo cual debe ser un aspecto a considerar en futuras investigaciones. 
La asociación existente entre la mejora en la agilidad con respecto al incremento en la memoria de los sujetos principiantes, respalda la tendencia a asociar los cambios a nivel físico con los cambios a nivel cognitivo comentados por Sanabria (1995).

Por otro lado se discrepa con Morel (1996), que afirma que la agilidad no es una buena predictora del estado cognoscitivo, pues en la presente investigación sí se halló una relación positiva, por lo cual se estimula a profundizar en el estudio de esta relación.

En la presente investigación las correspondientes correlaciones se determinaron con los respectivos porcentajes de cambio presentados por los participantes, con lo cual se puede afirmar que las relaciones se deben a los cambios producidos durante el tratamiento y no a situaciones ajenas al mismo, por lo cual se sugiere seguir este procedimiento en futuros análisis de esta índole.

Los incrementos en las facultades físicas y cognitivas repercutieron en la capacidad para desarrollar las diferentes actividades de la vida diaria de las adultas mayores participantes, pues se debe recordar que con "pequeños cambios en la capacidad fisiológica se obtienen substanciales efectos en el rendimiento de los adultos mayores" (Buchner, Larson, Wagner, Koepsell, y Lateur, 1996). Estos efectos se corroboraron mediante la interacción establecida entre el investigador y las participantes, las cuales externaron haber adquirido una mayor facilidad para movilizarse y realizar diferentes actividades del hogar y del cuidado personal; lo cual es congruente con lo encontrado por Ballestero (1999), que describió cómo luego de que un grupo de adultas mayores había participado de un programa de Karate Do, habían experimentado aumentos en la fuerza y en la flexibilidad, además de un cambio positivo en su facilidad para movilizarse dentro del hogar, subir y bajar gradas y alcanzar objetos ubicados a cierta altura dentro del hogar. Sin embargo no se contó con una medida más objetiva de estos efectos, por lo que se recomienda, en futuros estudios, incorporar la medición de cambios en la funcionalidad en actividades del diario vivir.

Basándose en la evidencia analizada se recomienda la difusión de la práctica del taekwondo en la población adulta mayor. Sin embargo, por las características de esta disciplina deportiva, es esencial la preparación que posea el profesor a cargo, el cual debe tener un amplio conocimiento de las características de esta población, de la actividad física y de la enseñanza del taekwondo, para así lograr desarrollar durante las prácticas, las actividades adecuadas que produzcan los múltiples beneficios que puede brindar esta disciplina a los adultos mayores.

\section{REFERENCIAS}

Aguirre, L. (2000). Efectos crónicos del entrenamiento de fuerza sobre procesos cognitivos en personas mayores de 60 años. Tesis de grado para optar por el grado de Licenciatura en Educación Física con énfasis en Salud. Universidad Nacional. Heredia, Costa Rica.

Ballestero, C (1999). Modificaciones del estado anímico, calidad de vida y autoestima en adultas mayores por medio de un programa de actividad sistemática del Karate Do. Tesis de grado para optar por el grado de Magister Scientiae en Gerontología. Universidad de Costa Rica. San José, Costa Rica.

Becerro, M., Frontera, W., y Santoja, R. (1999). La salud y la actividad física en las personas mayores. Editorial Santoja, Madrid.

Beissner, K., Collins, J., y Holmes, H. (2000). Muscle force and range of motion as predictors of function in older adults. Physical Therapy. 80 (6) $556-563$.

Biddle, S. (1995). Exercise and psychosocial Health. Research Quarterly for Exercise and Sport. 66 (4), 292-297.

Buchner, D., Larson, E., Wagner, E., Koepsell, T y Lateur, B. (1996). Evidence for a non-linear relationship between leg strength and gait speed. Age and aging. 25, (5), 386-39.

Carazo, P., Ballestero, C., y Salazar, W. (2000). Efectos del entrenamiento y desentrenamiento en la fuerza, flexibilidad, estado anímico y autoestima de adultos mayores por medio de un programa de artes marciales. Memoria VII Simposio Internacional en Ciencias del Deporte y la Salud. San José, Costa Rica. 
Clark, B. (1989). Test for fitness in older adults. Joperd. 60 (3) $66-71$.

Etnier, L., Salazar, W., Landers, D., Petruzzello, S., Han, M., y Nowell, P. (1997). The influence of physical fitness and exercise upon cognitive fuctioning : meta-analysis. Journal of Sport \& Exercise Psychology. 19249 - 277.

Hess, M., y Blanchard, F. (1999). Social cognition and aging. Academic Press. California.

Hirvensalo, M., Rantanen, T., y Heikkinen, E. (2000). Mobility difficulties and physical activity as predictors of mortality and loss of independence in the community-living older population. Journal of the American Geriatrics Society 48 (5) 493-498.

Jones, J., Rikli, R., y Beam , W. (1999). A 30s chair stand test as measure of lower body strength in community - residing older adults. Research Quarterly for Exercise and Sport 70 (2) 113 119.

Marqués, R. (1993). Vivir la vejez. Barcelona, España. Editorial Indigo.

Meléndez, A. (2000). Actividades físicas para mayores. Madrid, España. Editorial Gymnos.

Méndez, E. (1998). Calidad de vida de las personas mayores residentes en el Hogar para Ancianos “Alfredo y Delia Gonzáles Flores”. Estudio de caso. Tesis de grado para optar por el grado de Magister Scientiae en Gerontología. Universidad de Costa Rica. San José, Costa Rica.

Mora, M. (2002). Perspectiva subjetiva de la calidad de vida del adulto mayor ${ }_{2}$ diferencias ligadas al género, edad y a la práctica de actividad físico recreativa en 21 centros diurnos costarricenses. Tesis de grado para optar por el grado de Magister Scientiae en Salud Integral y Movimiento Humano con énfasis en Salud. Universidad Nacional. Heredia, Costa Rica.

Morel, V. (1996). Relaciones entre la funcionabilidad, el nivel de actividad física, el estado cognitivo y anímico de adultos mayores participando en programas recreativos de actividad física. Memoria III Simposio Internacional en Ciencias del Deporte y la Salud. San José, Costa Rica.

Morel, V., y Salazar, W. (1995). Estudio descriptivo de la condición física, del estado anímico y cognoscitivo de adultos mayores del área de San José. Memoria II Simposio Internacional en Ciencias del Deporte y la Salud. San José, Costa Rica.

Morey M,. Pieper, C., y Coroni-Huntley, J. (1998). Physical fitness and functional limitations in comunity - dwelling older adults. Medicine \& Science In Sports \& Exercise. 30 (5), 715-723.

Nichols, J., Hitzelberger, L., Sherman, J., y Patterson, P. (1995). Effects of resistance training on muscular strength and functional abilities of comunity dwelling older adults. Journal of aging and physical activity. 3 (3) 238-250.
Rojas, M. (1997). Bienestar general subjetivo y depresión en ancianos enfermos crónicos: Un estudio de casos. Tesis de grado para optar por el grado de Magister Scientiae en Gerontología. Universidad de Costa Rica. San José, Costa Rica.

Saliba, D., Orlando, M., Wenger, N., Hays, R., y Rubenstein, L. (2000). Identifying a Short Functional Disability Screen for Older Persons. The Journals of Gerontology Series A: Biological Sciences and Medical Sciences 55:M750-M756.

Sanabria, I. (1995). Meta - Análisis sobre los efectos del ejercicio en parámetros cognitivos. Tesis de grado para optar por el grado Licenciada en Educación Física. Universidad de Costa Rica. San José, Costa Rica.

Shephard, R. (1995). Physical Activity, Health, and different Life Stages. Research Quarterly for Exercise and Sport. 66 (4), 298-302.

Solera, A. (2000). Efectos de la deshidratación y la rehidratación en variables relacionadas con el rendimiento deportivo. Tesis de grado para optar por el grado de Magister Scientiae en Ciencias del Movimiento Humano. Universidad de Costa Rica. San José, Costa Rica.

Starr,J., Deary, I., Inch, S., Cross, S., y MacLennan, W. (1997). Age-associated cognitive decline in healthy old people. Age and Ageing. 26, (4) 295-300.

Timiras, P. (1997). Bases fisiológicas del envejecimiento y geriatría. Editorial Masson. Barcelona.

Weicker, H (1996). Biochemical metabolic and muscular adaptation by continuos physical activities at an older age. Deutsche Zeitschrift fuer sport medizin. 47 (5) 240-248

Yadory, O. (1997). Artes Marciales y defensa personal. Editores Mexicanos Unidos, México. 
Revista de Ciencias del Ejercicio y la Salud ${ }^{\circledR}$ Vol. 2, No 1, 2002 